Glasgow Math. J. 47 (2005) 461-469. (C) 2005 Glasgow Mathematical Journal Trust. doi:10.1017/S0017089505002703. Printed in the United Kingdom

\title{
GAPS OF OPERATORS, II
}

\author{
IL BONG JUNG, MI RYEONG LEE and PIL SANG LIM \\ Department of Mathematics, Kyungpook National University, Daegu, 702-701, Korea \\ e-mail:ibjung@knu.ac.kr,lmr67@yumail.ac.kr,limath@hanmail.net
}

(Received 9 November, 2004; accepted 1 July, 2005)

\begin{abstract}
In [11] the authors obtained an operator matrix with two variables that distinguishes the classes of $p$-hyponormal operators, $w$-hyponormal, absolute$p$-paranormal, and normaloid operators on Hilbert spaces. We establish the general model for $n$ variables, which provides many more examples to show that such classes are distinct.
\end{abstract}

2000 Mathematics Subject Classification. 47B20, 47B37, 47A15.

1. Introduction. Let $\mathcal{H}$ be a complex Hilbert space and let $\mathcal{L}(\mathcal{H})$ be the algebra of all bounded linear operators on $\mathcal{H}$. There are several classes of operators with weaker conditions than quasinormality; for example, $p$-hyponormal, $w$-hyponormal, p-quasihyponormal, absolute $p$-paranormal, $p$-paranormal, and normaloid operators, etc. Here is a brief review of those operators (see [5], [6], [10], and [12] for further discussion).

- An operator $T \in \mathcal{L}(\mathcal{H})$ is p-hyponormal if $\left(T^{*} T\right)^{p} \geq\left(T T^{*}\right)^{p}(0<p<\infty)$.

- $T$ is $\infty$-hyponormal if $\left(T^{*} T\right)^{p} \geq\left(T T^{*}\right)^{p}$, for all $p \in(0, \infty)$.

- $T$ is $p$-quasihyponormal if $T^{*}\left(\left(T^{*} T\right)^{p}-\left(T T^{*}\right)^{p}\right) T \geq 0(0<p<\infty)$.

- $T$ is an $A(p)$-operator if $\left(T^{*}|T|^{2 p} T\right)^{1 /(p+1)} \geq|T|^{2}(0<p<\infty)$.

- $T$ is p-paranormal if $\left\||T|^{p} U|T|^{p} x\right\| \geq\left\||T|^{p} x\right\|^{2}$ for all unit vectors $x \in \mathcal{H}(0<$ $p<\infty)$, where $U$ is the partially isometric part of the polar decomposition of $T$. In particular, 1-paranormality is referred to as paranormality.

- $T$ is absolute-p-paranormal if $\left\||T|^{p} T x\right\| \geq\|T x\|^{p+1}$ for all unit vectors $x \in \mathcal{H}$ $(0<p<\infty)$. Note that absolute-1-paranormality and 1-paranormality are equivalent.

- $T$ is $w$-hyponormal if $|\widetilde{T}| \geq|T|$, where $\widetilde{T}:=|T|^{1 / 2} U|T|^{1 / 2}$ is the Aluthge transform of $T$ ([2], [3], [9]).

- $T$ is normaloid if $\|T\|=r(T)$, where $r(T)$ is the spectral radius of $T$, which is equivalent to $\left\|T^{n}\right\|=\|T\|^{n}$ for all natural numbers $n$. (See [6, p. 100].)

There are several well-known relationships among the classes of operators described above. The interesting implications in this note are as follows:

- quasinormal $\Rightarrow p$-hyponormal $\Rightarrow p$-quasihyponormal $\Rightarrow A(p)$-operator $\Rightarrow$ absolute- $p$-paranormal $\Rightarrow$ normaloid.

Only a few examples of these operators, in particular $p$-hyponormal operators, have been known, and so it is worthwhile to develop examples to show that these classes are distinct. For this purpose, in [11] the authors considered a matrix of block operators with 2 variables and obtained a graph to classify those classes in 2-dimensional space. In this note we extend the study of the 2 variable version in [11] to yield the general version of $n$ variables which establishes some examples to show that the classes of 
$p$-hyponormal operators are distinct for each $n \geq 2$. Thus such examples are abundant! In addition, we consider the graphs for operators. These will be discussed in this note in 3-dimensional space.

2. p-hyponormality. Let $C=\left(c_{i j}\right)$ be an $n \times n$ matrix with $c_{i j}=1 / n(1 \leq i, j \leq n)$ and let $D \equiv D\left(x_{1}, x_{2}, \ldots, x_{n}\right):=\operatorname{Diag}\left\{x_{1}, x_{2}, \ldots, x_{n}\right\}$ with $x_{i} \geq 0, i=1, \ldots, n$. We define $T\left(x_{1}, x_{2}, \ldots, x_{n}\right)$ on $\mathcal{H} \equiv \mathbb{C}^{n} \otimes \ell_{2}(\mathbb{Z})$ by

$$
T\left(x_{1}, x_{2}, \ldots, x_{n}\right)=\left(\begin{array}{llllllll}
\ddots & & & & & & \\
\ddots & O & & & & & \\
& C & O & & & & \\
& & C & O & & & \\
& & & D & O & & \\
& & & & D & O & \\
& & & & & \ddots & \ddots
\end{array}\right),
$$

where $\cdot$ denotes the center of the two sided infinite matrix.

THEOREM 2.1. Let $p \in(0, \infty)$. Then the following assertions are equivalent:

(i) $T\left(x_{1}, x_{2}, \ldots, x_{n}\right)$ is p-hyponormal;

(ii) $n \geq\left(1 / x_{1}\right)^{2 p}+\cdots+\left(1 / x_{n}\right)^{2 p}$ with $x_{i}>0,1 \leq i \leq n$.

The following lemma proves Theorem 2.1.

LEMmA 2.2. Let $p \in(0, \infty)$. Then the following assertions are equivalent:

(i) $T\left(x_{1}, x_{2}, \ldots, x_{n}\right)$ is p-hyponormal;

(ii) $\Delta_{k}>0(1 \leq k \leq n-1)$ and $\Delta_{n} \geq 0$, where

$$
\begin{aligned}
& \Delta_{1}:=x_{1}^{2 p}-\frac{1}{n} \text { and } \\
& \Delta_{k}:=-\frac{1}{n} \sum_{1 \leq i \leq k}\left(\prod_{1 \leq j \leq k, j \neq i} x_{j}^{2 p}\right)+\prod_{1 \leq i \leq k} x_{i}^{2 p} \quad(2 \leq k \leq n) .
\end{aligned}
$$

Proof. First note that $T\left(x_{1}, x_{2}, \ldots, x_{n}\right)$ is $p$-hyponormal if and only if $D^{2 p}-C \geq 0$. Let

$$
A:=D^{2 p}-C=\left(\begin{array}{rrrr}
x_{1}^{2 p}-\frac{1}{n} & -\frac{1}{n} & \cdots & -\frac{1}{n} \\
-\frac{1}{n} & x_{2}^{2 p}-\frac{1}{n} & \cdots & -\frac{1}{n} \\
\vdots & \vdots & \ddots & \vdots \\
-\frac{1}{n} & -\frac{1}{n} & \cdots & x_{n}^{2 p}-\frac{1}{n}
\end{array}\right)
$$


and let $S_{k}$ be the $k \times k$ matrix in the upper left corner of $A$. Then the determinant of the matrix $S_{k}, 2 \leq k \leq n$, is

$$
\Delta_{k}:=-\frac{1}{n} \sum_{1 \leq i \leq k}\left(\prod_{1 \leq j \leq k, j \neq i} x_{j}^{2 p}\right)+\prod_{1 \leq i \leq k} x_{i}^{2 p} .
$$

According to the Nested Determinants Test (cf. [4, p. 213]), obviously (ii) implies (i). We shall show that (i) implies (ii) here. If $D^{2 p}-C \geq 0$, then obviously $S_{k} \geq 0$ and

$$
x_{i} \neq 0 \text { for } i=1,2, \ldots, n .
$$

Suppose that $\Delta_{k}=0$ for $1 \leq k<n$. Then $\Delta_{k+1}=\cdots=\Delta_{n}=0$ (cf. [4, Proposition 2.6]). Multiplying by $x_{k+1}^{2 p}$ and adding $-\frac{1}{n} \prod_{1 \leq i \leq k} x_{i}^{2 p}$ to (2.2), we have

$$
\Delta_{k+1}=\Delta_{k} x_{k+1}^{2 p}-\frac{1}{n} \prod_{1 \leq i \leq k} x_{i}^{2 p}=0 \quad(2 \leq k<n) .
$$

Hence $x_{1}^{2 p} \cdots x_{k}^{2 p}=0$. Therefore $x_{k_{0}}=0$ for some $2 \leq k_{0} \leq k$, which contradicts (2.3).

Proof of Theorem 2.1. The implication (i) $\Rightarrow$ (ii) follows easily from Lemma 2.2. For the reverse implication (ii) $\Rightarrow$ (i), we assume $n \geq\left(1 / x_{1}\right)^{2 p}+\cdots+\left(1 / x_{n}\right)^{2 p}$ with $x_{i}>0,1 \leq i \leq n$. Then

$$
n>\left(\frac{1}{x_{1}}\right)^{2 p}+\cdots+\left(\frac{1}{x_{k}}\right)^{2 p}, \quad(1 \leq k \leq n-1) .
$$

Hence we may obtain $\Delta_{k}>0(1 \leq k \leq n-1)$ by a simple computation. Since $\Delta_{n} \geq 0$ is equivalent to $n \geq\left(1 / x_{1}\right)^{2 p}+\cdots+\left(1 / x_{n}\right)^{2 p}$, we have proved this theorem.

For $0<p \leq \infty$, we denote by $\mathcal{R}_{p}^{(n)}$ the set of $\left(x_{1}, \ldots, x_{n}\right)$ in $\mathbb{R}_{+}^{(n)}$ such that $T\left(x_{1}, x_{2}, \ldots, x_{n}\right)$ is $p$-hyponormal, where $\mathbb{R}_{+}$is the set of nonnegative real numbers. The following proposition shows that the classes of $p$-hyponormal operators are distinct for $0<p \leq \infty$.

Proposition 2.3. For $0<q<p<\infty, \mathcal{R}_{\infty}^{(n)} \varsubsetneqq \mathcal{R}_{p}^{(n)} \varsubsetneqq \mathcal{R}_{q}^{(n)}$.

Proof. Since the case of $n=2$ was proved in [11], we may assume that $n \geq 3$. Let us consider $x_{1}=x_{2}=\cdots=x_{n-2}=1$ and let

$$
\left.\mathcal{R}_{p}^{(n)}\right|_{\left(1, \ldots, 1, x_{n-1}, x_{n}\right)}:=\left\{(\underbrace{1, \ldots, 1}_{(n-2)}, x_{n-1}, x_{n}): 2 \geq\left(\frac{1}{x_{n-1}}\right)^{2 p}+\left(\frac{1}{x_{n}}\right)^{2 p}\right\},
$$

which is the projection of $\mathcal{R}_{p}^{(n)}$ w.r.t. $x_{1}=x_{2}=\cdots=x_{n-2}=1$. Then a computation similar to that in the proof of [11, Lemma 2.2] shows that $\left.\mathcal{R}_{p}^{(n)}\right|_{\left(1, \ldots, 1, x_{n-1}, x_{n}\right) \varsubsetneqq} \varsubsetneqq$ $\left.\mathcal{R}_{q}^{(n)}\right|_{\left(1, \ldots, 1, x_{n-1}, x_{n}\right)}$. Obviously $\mathcal{R}_{p}^{(n)} \varsubsetneqq \mathcal{R}_{q}^{(n)}$.

REMARK 2.4. Let $\mathcal{R}_{\mathrm{qn}}^{(n)}$ be the set of $\left(x_{1}, \ldots, x_{n}\right) \in \mathbb{R}_{+}^{(n)}$ for which the operator $T\left(x_{1}, x_{2}, \ldots, x_{n}\right)$ is quasinormal. Then by the definition of quasinormality, we may 
show that $\mathcal{R}_{\mathrm{qn}}^{(n)}$ is the singleton $\{(1, \ldots, 1)\}$. This fact points out an error in $[\mathbf{1 1}$, Theorem $2.3(\mathrm{v})]$.

Proposition 2.5. For $p \in(0, \infty)$, we have the following equalities.

(i) $\mathcal{R}_{\infty}^{(n)}=[1, \infty) \times \cdots \times[1, \infty)(n-$ copies $)$,

(ii) $\bigcup_{p>0} \mathcal{R}_{p}^{(n)}=\left\{\left(x_{1}, \ldots, x_{n}\right): x_{1} x_{2} \cdots x_{n}>1\right\} \cup\{(1, \ldots, 1)\}$.

Proof. (i) Let $T\left(x_{1}, \ldots, x_{n}\right)$ be $\infty$-hyponormal. Then by (2.1), we have $x_{i}^{2 p} \geq \frac{1}{n}$ for all $p>0$ and $i=1, \ldots, n$. Hence $x_{i} \geq \lim _{p \rightarrow \infty}\left(\frac{1}{n}\right)^{\frac{1}{2 p}}=1$.

Conversely, suppose $x_{i} \geq 1(1 \leq i \leq n)$. By (2.1) and (2.2), we may show easily that $I_{n} \geq C$, where $I_{n}$ is the $n \times n$ identity matrix. Hence $D^{2 p} \geq I_{n} \geq C$ for all $p \in(0, \infty)$, which proves that $T\left(x_{1}, \ldots, x_{n}\right)$ is $p$-hyponormal. Then

(ii) Let $\left(x_{1}, \ldots, x_{n}\right) \in \mathcal{R}_{p}^{(n)}$ for some $p>0$ and suppose $x_{i} \neq 1$ for some $i=1, \ldots, n$.

$$
n \cdot x_{1}^{2 p} x_{2}^{2 p} \cdots x_{n}^{2 p} \geq \sum_{i=1}^{n} \prod_{1 \leq j \neq i \leq n} x_{j}^{2 p} \geq n \cdot \sqrt[n]{\left(x_{1} x_{2} \cdots x_{n}\right)^{2 p(n-1)}},
$$

which shows that $x_{1} x_{2} \cdots x_{n} \geq 1$. Now suppose that $x_{1} x_{2} \cdots x_{n}=1$. According to (2.4), we have

$$
n=\left(\frac{1}{x_{1}}\right)^{2 p}+\left(\frac{1}{x_{2}}\right)^{2 p}+\cdots+\left(\frac{1}{x_{n}}\right)^{2 p}
$$

Hence $x_{1}=\cdots=x_{n}=1$ (use the relationship of arithmetic and geometric means). This contradiction shows that $x_{1} x_{2} \cdots x_{n}>1$. Hence $\left(x_{1}, \ldots, x_{n}\right)$ belongs to the set on the right side in (ii).

On the other hand, since $(1, \ldots, 1) \in \mathcal{R}_{p}^{(n)}$ for every $p>0$, we may suppose that $x_{1} x_{2} \cdots x_{n}>1$ to prove the reverse inclusion. Let us define

$$
\varphi(p)=\left(\frac{1}{x_{1}}\right)^{2 p}+\left(\frac{1}{x_{2}}\right)^{2 p}+\cdots+\left(\frac{1}{x_{n}}\right)^{2 p} .
$$

Then a simple computation shows that $\lim _{p \rightarrow 0^{+}} \varphi^{\prime}(p)=-2 \ln \left(x_{1} \cdots x_{n}\right)<0$, and hence $\varphi^{\prime}(p)<0$ on $\left(0, p_{0}\right)$ for sufficiently small $p_{0}>0$. Since $\varphi(0)=n$, we have

$$
n>\left(\frac{1}{x_{1}}\right)^{2 p}+\left(\frac{1}{x_{2}}\right)^{2 p}+\cdots+\left(\frac{1}{x_{n}}\right)^{2 p}
$$

for such $p \in\left(0, p_{0}\right)$. Thus by Theorem $2.1,\left(x_{1}, \ldots, x_{n}\right) \in \cup_{0<p<p_{0}} \mathcal{R}_{p}^{(n)}$.

We consider the graph of the set $\mathcal{R}_{p}^{(3)}$ for $p$-hyponormal operators.

EXAmple 2.6 (The case $n=3$ ). It follows from Theorem 2.1 that $T(x, y, z)$ is $p$ hyponormal if and only if $3 \geq(1 / x)^{2 p}+(1 / y)^{2 p}+(1 / z)^{2 p}(x, y, z>0)$. The regions for the $p$-hyponormality of $T(x, y, z)$ are shown in Figure 2.1 .

3. Absolute p-paranormality. By direct computation, we obtain the following lemma. 


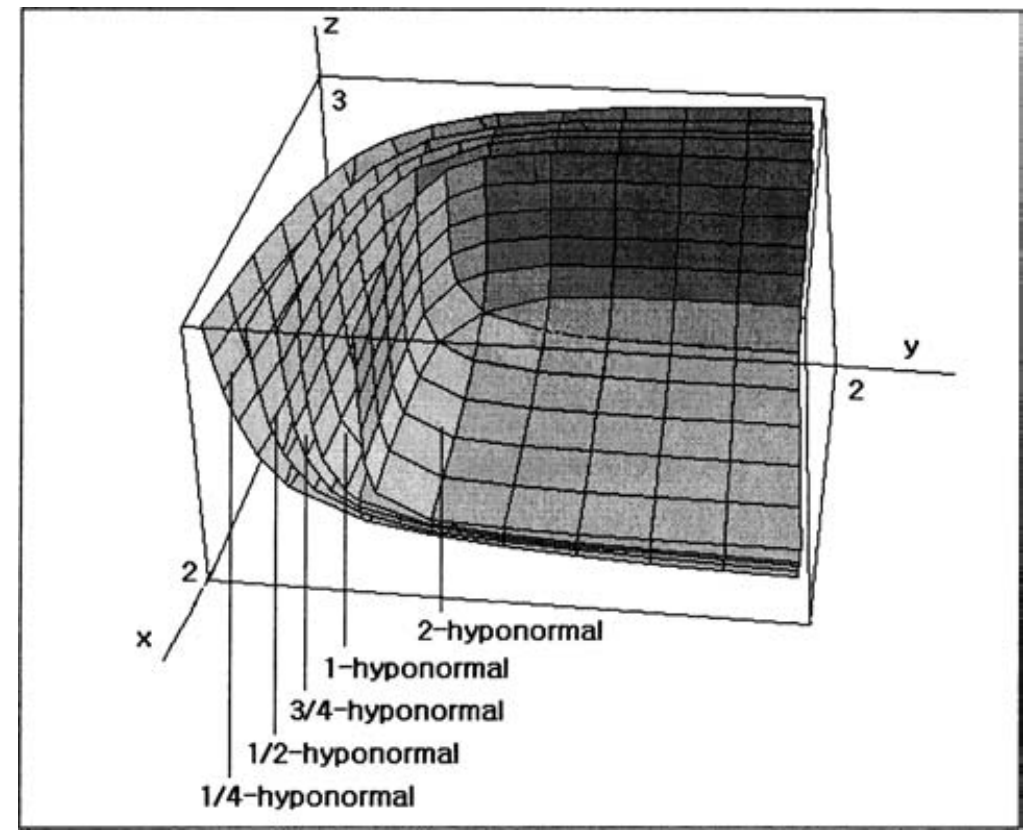

Figure 2.1

LEMMA 3.1. Let $B=\left(b_{i j}\right)$ be an $n \times n$ matrix satisfying $b_{i j}=a+b($ if $i=j)$ and $b_{i j}=a($ if $i \neq j)$, where $a, b \in \mathbb{R}$. Then $\operatorname{det} B=b^{n-1}(n a+b)$ for all $n \geq 1$.

THEOREM 3.2. For any $p>0$, the following assertions are equivalent:

(i) $T\left(x_{1}, x_{2}, \ldots, x_{n}\right)$ is absolute p-paranormal;

(ii) $x_{1}^{2 p}+x_{2}^{2 p}+\cdots+x_{n}^{2 p} \geq n$ and $x_{i} \geq 0(1 \leq i \leq n)$.

Proof. For brevity, let us put $T:=T\left(x_{1}, x_{2}, \ldots, x_{n}\right)$. It is well known ([6, Theorem 1, p. 174] and its proof) that $T$ is absolute $p$-paranormal if and only if for all $\lambda>0$, we have

$$
\Theta(\lambda):=T^{*}\left(T^{*} T\right)^{p} T-(p+1) \lambda^{p}\left(T^{*} T\right)+p \lambda^{p+1} \geq 0 .
$$

Obviously we have

$$
\begin{aligned}
\Theta(\lambda)= & \operatorname{Diag}\left\{\ldots, C^{2 p+2}, C^{2 p+2}, C D^{2 p} C, D^{2 p+2}, D^{2 p+2}, D^{2 p+2}, \ldots\right\} \\
& -(p+1) \lambda^{p} \operatorname{Diag}\left\{\ldots, C^{2}, C^{2}, D^{2}, D^{2}, D^{2}, \ldots\right\}+p \lambda^{p+1} .
\end{aligned}
$$

To show that $\Theta(\lambda) \geq 0$, for all $\lambda>0$, we need to prove that

(i) $\Theta_{1}(\lambda):=C^{2 p+2}-(p+1) \lambda^{p} C^{2}+p \lambda^{p+1} \geq 0(\lambda>0)$,

(ii) $\Theta_{2}(\lambda):=C D^{2 p} C-(p+1) \lambda^{p} C^{2}+p \lambda^{p+1} \geq 0(\lambda>0)$,

(iii) $\Theta_{3}(\lambda):=D^{2 p+2}-(p+1) \lambda^{p} D^{2}+p \lambda^{p+1} \geq 0(\lambda>0)$.

Let $\Delta_{k}^{(i)}$ denote the determinant of the $k \times k$ submatrix of the upper left corner in $\Theta_{i}(\lambda)$ for each $i=1,2,3$ and $k=1, \ldots, n$. In order to obtain $\Theta_{i}(\lambda) \geq 0$ for all $\lambda>0$, it is sufficient to show that $\Delta_{n}^{(i)} \geq 0$ and $\Delta_{k}^{(i)}>0$ for $k=1, \ldots, n-1$. 
First, by a simple calculation, we have the $n \times n$ matrix $\Theta_{1}(\lambda)=\left[\theta_{i j}^{(1)}\right]$, where

$$
\theta_{i j}^{(1)}= \begin{cases}\frac{1-(p+1) \lambda^{p}}{n}+p \lambda^{p+1} & \text { for } \quad(i=j) \\ \frac{1-(p+1) \lambda^{p}}{n} & \text { for } \quad(i \neq j)\end{cases}
$$

and $i, j=1,2, \ldots, n$. Using Lemma 3.1, we have for $k=1,2, \ldots, n$,

$$
\Delta_{k}^{(1)}=\left(p \lambda^{p+1}\right)^{k-1}\left(\frac{k}{n}-\frac{k}{n}(p+1) \lambda^{p}+p \lambda^{p+1}\right) .
$$

Put $f_{1}(\lambda):=p \lambda^{p+1}-\frac{k}{n}(p+1) \lambda^{p}+\frac{k}{n}$, for $k=1,2, \ldots, n$. Then, $f_{1}(\lambda)$ has its minimum value at $\lambda=\frac{k}{n}$ and $f_{1}\left(\frac{k}{n}\right)=\frac{k}{n}\left(1-\left(\frac{k}{n}\right)^{p}\right)$ for $k=1,2, \ldots, n$. Since $\Delta_{k}^{(1)}=\left(p \lambda^{p+1}\right)^{k-1}$. $f_{1}(\lambda)$, we have that $\Delta_{k}^{(1)}>0$ for $k=1,2, \ldots, n-1$ and $\Delta_{n}^{(1)} \geq 0$, and so $\Theta_{1}(\lambda) \geq 0$ $(\lambda>0)$.

Consider also the matrix $\Theta_{3}(\lambda)=\left[\theta_{i j}^{(3)}\right]$, where

$$
\theta_{i j}^{(3)}= \begin{cases}x_{i}^{2 p+2}-(p+1) \lambda^{p} x_{i}^{2}+p \lambda^{p+1} & \text { for } \quad(i=j) \\ 0 & \text { for } \quad(i \neq j)\end{cases}
$$

and $i, j=1,2, \ldots, n$. Put $f_{3}(\lambda):=x_{i}^{2 p+2}-(p+1) \lambda^{p} x_{i}^{2}+p \lambda^{p+1}$ for $i=1,2, \ldots, n$. Then, by the same method, $f_{3}(\lambda)$ has its minimum value at $\lambda=x_{i}^{2}$ and $f_{3}\left(x_{i}^{2}\right)=0$ for $i=1,2, \ldots, n$. Therefore, $\Theta_{3}(\lambda) \geq 0$ for all $\lambda>0$.

Next, by another calculation, we have the matrix $\Theta_{2}(\lambda)=\left[\theta_{i j}^{(2)}\right]$, where

$$
\theta_{i j}^{(2)}= \begin{cases}\frac{x_{1}^{2 p}+\cdots+x_{n}^{2 p}-n(p+1) \lambda^{p}}{n^{2}}+p \lambda^{p+1} & \text { for } \quad(i=j) \\ \frac{x_{1}^{2 p}+\cdots+x_{n}^{2 p}-n(p+1) \lambda^{p}}{n^{2}} & \text { for } \quad(i \neq j)\end{cases}
$$

and $i, j=1,2, \ldots, n$. Using Lemma 3.1, we have for $k=1,2, \ldots, n$,

$$
\Delta_{k}^{(2)}=\left(p \lambda^{p+1}\right)^{k-1}\left[\frac{k}{n^{2}}\left(\sum_{i=1}^{n} x_{i}^{2 p}-n(p+1) \lambda^{p}\right)+p \lambda^{p+1}\right] .
$$

Put

$$
f_{2}(\lambda):=p \lambda^{p+1}-\frac{k}{n}(p+1) \lambda^{p}+\frac{k}{n^{2}} \sum_{i=1}^{n} x_{i}^{2 p},
$$

for $k=1,2, \ldots, n$. Then, we can see that $f_{2}(\lambda)$ has its minimum value at $\lambda=\frac{k}{n}$. If $x_{1}^{2 p}+\cdots+x_{n}^{2 p} \geq n$, then

$$
f_{2}\left(\frac{k}{n}\right)=\frac{k}{n^{2}} \cdot \sum_{i=1}^{n} x_{i}^{2 p}-\left(\frac{k}{n}\right)^{p+1} \geq \frac{k}{n}\left[1-\left(\frac{k}{n}\right)^{p}\right]>0
$$

for $k=1,2, \ldots, n-1$. Since $f_{2}\left(\frac{n}{n}\right)=\frac{1}{n} \sum_{i=1}^{n} x_{i}^{2 p}-1 \geq 0$, the inequality $x_{1}^{2 p}+\cdots+$ $x_{n}^{2 p} \geq n$ implies that $\Theta_{2}(\lambda) \geq 0(\lambda>0)$. The reverse implication is obvious. 
Proposition 3.3. Let

$$
\Omega_{p}=\left\{\left(x_{1}, \ldots, x_{n}\right) \in \mathbb{R}_{+}^{(n)}: T\left(x_{1}, \ldots, x_{n}\right) \text { is absolute p-paranormal } .\right.
$$

Then

$$
\bigcap_{p>0} \Omega_{p}=\left\{\left(x_{1}, \ldots, x_{n}\right): x_{1} \cdots x_{n} \geq 1\right\}
$$

Proof. Let $\left(x_{1}, \ldots, x_{n}\right) \in \bigcap_{p>0} \Omega_{p}$. Then it follows from Theorem 3.2 that

$$
x_{1}^{2 p}+\cdots+x_{n}^{2 p} \geq n
$$

for $p>0$. Note that $x_{i}>0(1 \leq i \leq n)$ (because (3.1) holds for all $\left.p>0\right)$. Since

$$
x_{k} \geq\left(n-\sum_{1 \leq i \leq n, i \neq k} x_{i}^{2 p}\right)^{\frac{1}{2 p}}
$$

then, by letting $p \rightarrow 0$ in the inequality of (3.2), we have that

$$
x_{k} \geq \frac{1}{x_{1} \cdots x_{k-1} \cdot x_{k+1} \cdots x_{n}} .
$$

Conversely, consider the vector $\left(x_{1}, \ldots, x_{n}\right)$ in $\mathbb{R}_{+}^{(n)}$ satisfying $x_{1} \cdots x_{n} \geq 1$. Then

$$
\frac{x_{1}^{2 p}+\cdots+x_{n}^{2 p}}{n} \geq\left(x_{1}^{2 p} \cdots x_{n}^{2 p}\right)^{\frac{1}{n}} \geq 1 \text { for all } p>0
$$

and hence $x_{1}^{2 p}+\cdots+x_{n}^{2 p} \geq n$ for all $p>0$.

Proposition 3.4. For $p \in(0, \infty)$, the following assertions are equivalent:

(i) $T\left(x_{1}, \ldots, x_{n}\right)$ is absolute p-paranormal;

(ii) $T\left(x_{1}, \ldots, x_{n}\right)$ is p-quasihyponormal;

(iii) $T\left(x_{1}, \ldots, x_{n}\right)$ is p-paranormal;

(iv) $T\left(x_{1}, \ldots, x_{n}\right)$ is $A(p)$-operator;

(v) $x_{1}^{2 p}+\cdots+x_{n}^{2 p} \geq n\left(x_{i} \geq 0,1 \leq i \leq n\right)$.

In particular, $T\left(x_{1}, \ldots, x_{n}\right)$ is 1/2-paranormal if and only if $T\left(x_{1}, x_{2}, \ldots, x_{n}\right)$ is w-hyponormal.

Proof. For brevity, we denote $T=T\left(x_{1}, \ldots, x_{n}\right)$. Then, according to the definition of $p$-quasihyponormality, we have

$$
\begin{aligned}
T \text { is } p \text {-quasihyponormal } & \Longleftrightarrow T^{*}\left\{\left(T^{*} T\right)^{p}-\left(T T^{*}\right)^{p}\right\} T \geq 0 \\
& \Longleftrightarrow C D^{2 p} C-C=\left(\frac{1}{n} \sum_{i=1}^{n} x_{i}^{2 p}-1\right) C \geq 0 \\
& \Longleftrightarrow x_{1}^{2 p}+\cdots+x_{n}^{2 p} \geq n .
\end{aligned}
$$

Observe that $T$ is $p$-paranormal if and only if $U|T|^{p}=T\left(x_{1}^{p}, \ldots, x_{n}^{p}\right)$ is paranormal (i.e., absolute-1-paranormal), which is equivalent to the condition $x_{1}^{2 p}+\cdots+x_{n}^{2 p} \geq n$ 




Figure 3.2

by Theorem 3.2. Finally, a proof similar to that of Theorem 3.1 shows that assertions (iv) and (v) are equivalent.

COROLlARY 3.5. Let $\mathcal{N}(n)$ be the set of $\left(x_{1}, \ldots, x_{n}\right)$ in $\mathbb{R}_{+}^{(n)}$ such that $T\left(x_{1}, x_{2}, \ldots, x_{n}\right)$ is normaloid. Then

$$
\mathcal{N}(n) \backslash \bigcup_{p>0} \Omega_{p}=\underbrace{[0,1] \times \cdots \times[0,1]}_{(n)} \backslash(\underbrace{(1, \ldots, 1}_{(n)}) .
$$

Proof. Use the proof of [11, Proposition 2.8] and Proposition 3.4.

EXAmple 3.6. (Continued from Example 2.6). Recall that $T(x, y, z)$ is $p$ paranormal if and only if $x^{2 p}+y^{2 p}+z^{2 p} \geq 3$ and $x, y, z \geq 0$. The following Figure 3.2 shows the regions for $p$-paranormality.

REMARK 3.7. Let

$$
I \neq A=\left(\begin{array}{ll}
a & \bar{b} \\
b & c
\end{array}\right)
$$

be an otherwise arbitrary $2 \times 2$ positive matrix with entries of complex numbers. If $A^{2}=A$, then $A^{p}=A$ for all $p>0$. Hence

$$
A^{p}=A \text { for all } p>0 \Longleftrightarrow|b|^{2}=a(1-a) \& a+c=1 \text {. }
$$

(The case of $a=b=c=1 / 2$ is special.) If we take $a>0, c=1-a \geq 0$, and $b \in\left\{a(1-a) e^{i \theta}: 0 \leq \theta \leq 2 \pi\right\}$, by (3.4) we have that $T(x, y)$ is $p$-hyponormal if and 
only if

$$
y \geq\left(\frac{c\left(x^{2 p}-a\right)+|b|^{2}}{x^{2 p}-a}\right)^{\frac{1}{2 p}} \text { and } x>a^{\frac{1}{2 p}} .
$$

More generally, we may consider a $k \times k$ matrix $A=\left(a_{i j}\right)$. If we take an arbitrary $a_{i j}$ satisfying $A^{2}=A$ (which implies $A^{p}=A$ for any $p>0$ ), our technique introduced in this paper may provide a lot of examples to show that the classes of such operators discussed in this paper are distinct. We leave this work to the interested readers.

AcKnowledgment. The authors would like to express their gratitude to the referee for valuable comments that allowed us to improve the results herein. This work was supported by Korea Research Foundation Grant (KRF-2002-070-C00006).

\section{REFERENCES}

1. A. Aluthge, On p-hyponormal operators for $0<p<1$, Integral Equations Operator Theory 13 (1990), 307-315.

2. A. Aluthge and D. Wang, $w$-hyponormal operators, Integral Equations Operator Theory 36 (2000), 1-10.

3. A. Aluthge and D. Wang, w-hyponormal operators, II, Integral Equations Operator Theory 37 (2000), 324-331.

4. R. Curto and L. Fialkow, Recursively generated weighted shifts and the subnormal completion problem, Integral Equations Operator Theory 17 (1993), 202-246.

5. M. Fujii and Y. Nakatsu, On subclasses of hyponormal operators, Proc. Japan Acad. 51 (1975), 243-246. 2001).

6. T. Furuta, Invitation to linear operators (Taylor \& Francis Inc. London and New York,

7. T. Furuta, M. Ito and T. Yamazaki, A subclass of paranormal operators including class of log-hyponormal and several related classes, Sci. Math. 3 (1998), 389-403.

8. T. Huruya, A note on p-hyponormal operators, Proc. Amer. Math. Soc. 125 (1997), $3617-3624$.

9. M. Ito and T. Yamazaki, Relations between two inequalities $\left(B^{r / 2} A^{p} B^{r / 2}\right)^{r /(p+r)} \geq B^{r}$ and $A^{p} \geq\left(A^{p / 2} B^{r} A^{p / 2}\right)^{p /(p+r)}$ and their applications, Integral Equations Operator Theory $\mathbf{4 4}$ (2002), $442-450$.

10. I. Jung, E. Ko and C. Pearcy, Aluthge transforms of operators, Integral Equations Operator Theory 37 (2000), 437-448.

11. I. Jung, P. Lim and S. Park, Gaps of operators, J. Math. Anal. Appl. 304 (2005), 87-95.

12. S. Miyajima and I. Saito, $\infty$-hyponormal operators and their spectral properties, Acta Sci. Math. (Szeged) 67 (2001), 357-371.

13. D. Xia, Spectral theory of hyponormal operators (Birkhäuser Verlag, Boston, 1983). 